\title{
Comparison of Fusion Rates between Autologous Iliac Bone Graft and Calcium Sulfate with Laminectomy Bone Chips in Multilevel Posterolateral Spine Fusion
}

\author{
Meng-Ling Lu ${ }^{1 *}$, Tsung-Ting Tsai ${ }^{1,2 *}$, Lih-Huei Chen ${ }^{1}$, Po-Liang Lai ${ }^{1}$, Tsai-Sheng Fu ${ }^{1}$, \\ Chi-Chien Niu ${ }^{1}$, Wen-Jer Chen ${ }^{1 * \#}$ \\ ${ }^{1}$ Department of Orthopaedic Surgery, Spine Section, Chang Gung Memorial Hospital and Chang Gung University College \\ of Medicine, Taoyuan, Taiwan; ${ }^{2}$ Graduate Institute of Clinical Medical Sciences, Chang Gung University, Taoyuan, Taiwan. \\ Email: "chenwenj@adm.cgmh.org.tw
}

Received April 13 $3^{\text {th }}, 2013$; revised May $5^{\text {th }}, 2013$; accepted May 22 2013

Copyright (C) 2013 Meng-Ling Lu et al. This is an open access article distributed under the Creative Commons Attribution License, which permits unrestricted use, distribution, and reproduction in any medium, provided the original work is properly cited.

\begin{abstract}
Multilevel lumbar fusion usually requires a large quantity of iliac crest bone graft but the supply is usually insufficient, so an alternative bone graft substitute for autograft is needed. This prospective study investigated the efficacy of calcium sulfate by comparing the fusion rates between the experimental material (calcium sulfate pellets with bone chips from laminectomy) and autologous iliac bone graft in long segment (three-or four-level) lumbar and lumbosacral posterolateral fusion. Forty-five patients with degenerative scoliosis or spondylolisthesis received multilevel spine fusion and decompression. The experimental material of calcium sulfate pellets with decompression bone chips was placed on the experimental side and the iliac crest bone graft was placed on the control side. The fusion status was assessed radiographically at three-month intervals, and solid fusion was defined as a clear continuous intertransverse bony bridge at all levels. The average follow-up period was 34.4 months. Twenty-nine (64.4\%) patients showed solid fusion on the experimental side and $39(86.7 \%)$ patients on the control side. The overall fusion rate was $86.7 \%$. A statistically significant relation was found between the two sides with the Kappa coefficient of agreement of 0.436. Compared to the control side, the fusion rate of experimental side is significantly reduced $(p=0.014)$. The fusion ability of autograft is higher than the experimental material in multilevel lumbar posterolateral fusion. However, the overall fusion rate of calcium sulfate pellets is improved, compared with previously reported rates, which suggested that such material may be considered as an acceptable bone graft extender.
\end{abstract}

Keywords: Multilevel Spinal Fusion; Posterolateral Fusion; Calcium Sulfate; Bone Substitutes; Autologous Iliac Bone Graft

\section{Introduction}

Spine fusion is the mainstay technique for spinal stabilization, and posterolateral fusion has become a standard surgical technique for arthrodesis of lumbar spine [1,2]. Meanwhile, the use of autologous bone graft remains as the gold standard technique for posterolateral lumbar spinal fusion. Autologous bone graft is usually harvested from the iliac crest, and has osteogenic, osteoconductive, and osteoinductive properties. It provides calcium scaffolding for new bone formation and contains osteophytes for new bone growth. The advantages of autologous iliac bone graft include high fusion success rate, low risk of *Both authors contributed equally to this work and share the first authorship. \#Corresponding author. disease transmission, and histocompatibility [3].However, the harvesting of autologous bone graft is associated with a 8.6 percent rate for major complications and a 20.6 percent rate for minor complications, which includes increased operative time, donor site pain, blood loss, risk of infection, nerve damage and other potential complications [4,5]. Additionally, the supply of autologous iliac bone graft is usually limited, particularly for multilevel posterolateral spinal fusion.

The use of bone graft substitutes is the current development trend to overcome the problem of insufficient source of autograft and avoid the morbidity and potential complications of harvesting procedure. Bone graft substitutes can expand the existing quantity of autologous bone 
graft in posterolateral spinal fusion. Various bone raft substitutes and extenders have been examined in the past, such as allograft and a variety of biomaterials. However, allograft has performed disappointingly as a bone graft substitute with decreased chance of fusion in clinical trials of lumbar arthrodesis, and potential risk of disease transmission remains as the problem for allograft [6-8].

OsteoSet (Wright Medical Technologies, Arlington, Tenn.) bone graft pellets are made of medical grade calcium sulfate, which act as an osteoconductive matrix for ingrowth of blood vessels and osteogenic cells. The composition and crystalline structure of these calcium sulfate pellets were purposely designed so that the rate of absorption is consistent with the rate of new bone growth [9]. Previous studies have shown effective results of using calcium sulfate as bone graft extender in spinal arthrodesis $[10,11]$. However, no previous study has demonstrated its efficacy in multiple segment (three or four levels) posterolateral lumbar spinal fusion.

Current prospective research is focused on investigating the efficacy of calcium sulfate as alternative bone graft substitute by comparing the fusion rate between OsteoSet pellets mixed with local decompression bone chips and fresh autologous iliac crest bone in multilevel posterolateral lumbar and lumbosacral fusion.

\section{Materials and Methods}

Forty-five patients (13 men and 32 women), who required to receive three or four segments lumbar and lumbosacral spinal fusion with instrumentation and de compression procedure from October 1999 through May 2000, were included in this study. Each patient was well-explained and signed the informed consent before participating in this study. The diagnosis of inclusion criteria was degenerative scoliosis or spondylolisthesis, and exclusion criteria were revision spinal surgery, idiopathic scoliosis, spine tumor, and spine infection. The average age was 62.1 years old (range, $40-75$ years old), and the average follow-up period was 34.4 months (range, 31 38 months).

All patients had symptom of low back pain with intermittent claudication, and radiographic and magnetic resonance imaging (MRI) studies had revealed degenerative spondylolisthesis or degenerative scoliosis with lumbar spinal stenosis. At first, all patients received conservative treatment for several months, and then the surgical procedure of multilevel instrumented fusion with decompression was performed because there was no response to conservative treatment.

\subsection{Surgical Technique}

The standard posterior approach enabled the exposure of the posterior aspect of lumbar spine. According to the clinical symptoms and findings of MRI studies, canal decompression of posterior element was required and wide laminectomy was performed in all cases. Adjacent capsules and inter-and supraspinous ligaments were carefully preserved. Careful stripping of soft tissues and meticulous decortications of the transverse processes were performed at the posterolateral aspect of fused segments. The transpedicular instrumentation system was used for union reinforcement and spinal stabilization. Approximately $10 \mathrm{ml}$ of locally harvested bone obtained from the decompression site was morselized into small corticocancellous pieces and combined with about $20 \mathrm{ml}$ of OsteoSet pellets, and then this experimental material was randomly placed on either right or left side, which was labeled as the experimental side. On the contralateral side, approximately $20 \mathrm{ml}$ of autologous bone graft harvested from the posterior iliac crest was served as the control material, which was labeled as the control side. After operation, patients were able to walk within a week and wore hard corset for three months.

\subsection{Fusion Assessment}

The fusion status was radiographically assessed every three months (Figure 1) (Figure 2), and was graded by two independent orthopaedic surgeons who were blinded to the placement of the experimental material. "Fusion" was defined when a clear continuous intertransverse bony bridge at all levels was observed (Figure 3), and "Non-fusion" was graded when any suspected discontinuity at any fusion level or any gaps in the fusion mass was observed in the anteroposterior radiographs.

If the formation of new bone mass was apparent and solid fusion was defined on both sides of spine, the fusion masses were measured and compared between the experimental and control sides.

\subsection{Statistical Methods}

For the dependent outcomes at the experimental and control sides, we used generalized estimating equations (GEE) for logistic regression to estimate the odds ratio of fusion between the two sides. To measure the concordance between the two sides, Kappa coefficient of agreement was performed. Fisher's exact test was also used to compare the fusion rate in level $(3,4)$ and spine $(\mathrm{L}, \mathrm{S})$. The significant level alpha was 0.05 . All analyses were performed by SPSS statistics software version 17.0 (Chicago, IL).

\section{Results}

Twenty-nine (64.4\%) patients showed clear continuous intertransverse bony bridge at all levels on the experi- 


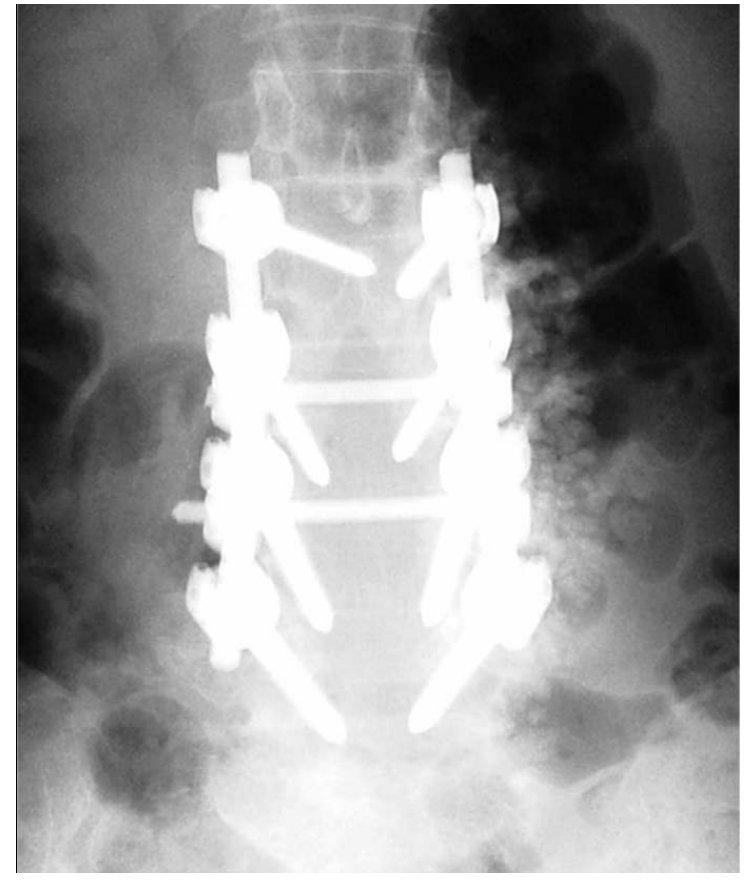

Figure 1. A 62-year-old female who received posterolateral spinal fusion from $\mathrm{L} 2$ to $\mathrm{L} 5$ for degenerative lumbar scoliosis and spinal stenosis. The Cotrel-Dubousset transpedicular instrumentation system was used. Locally harvested bone chips from the decompression site was combined with OsteoSet pellets and placed on the right side of spine; whereas, autologous bone graft harvested from posterior iliac crest was placed on the left side.

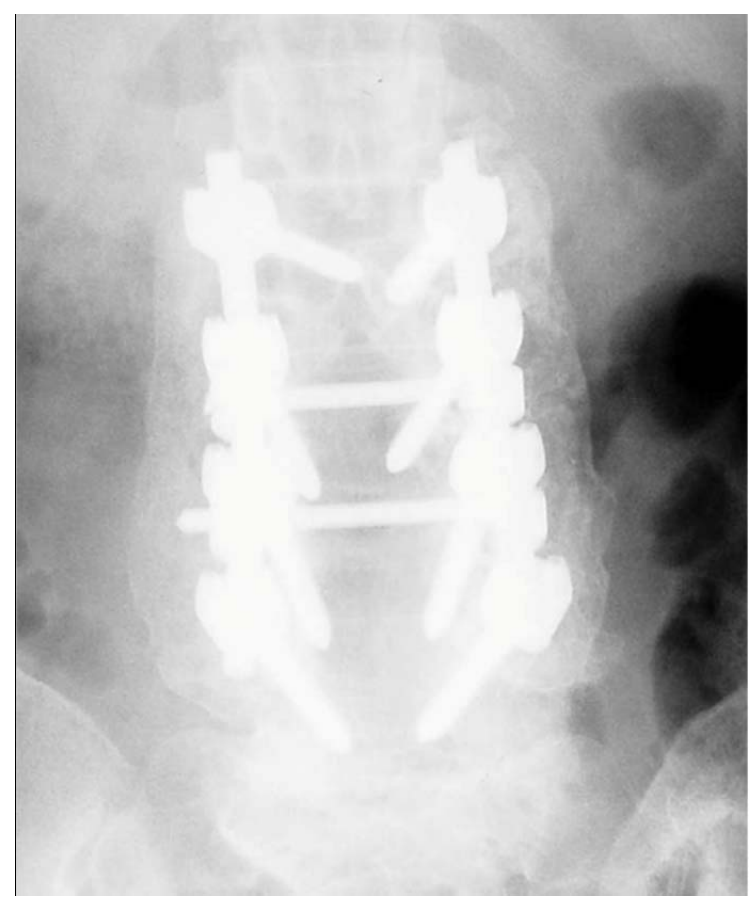

Figure 2. A radiograph at six-month follow-up showed that OsteoSet pellets were absorbed and an intertransverse bony bridge from L2 to L5 was observed on each side.

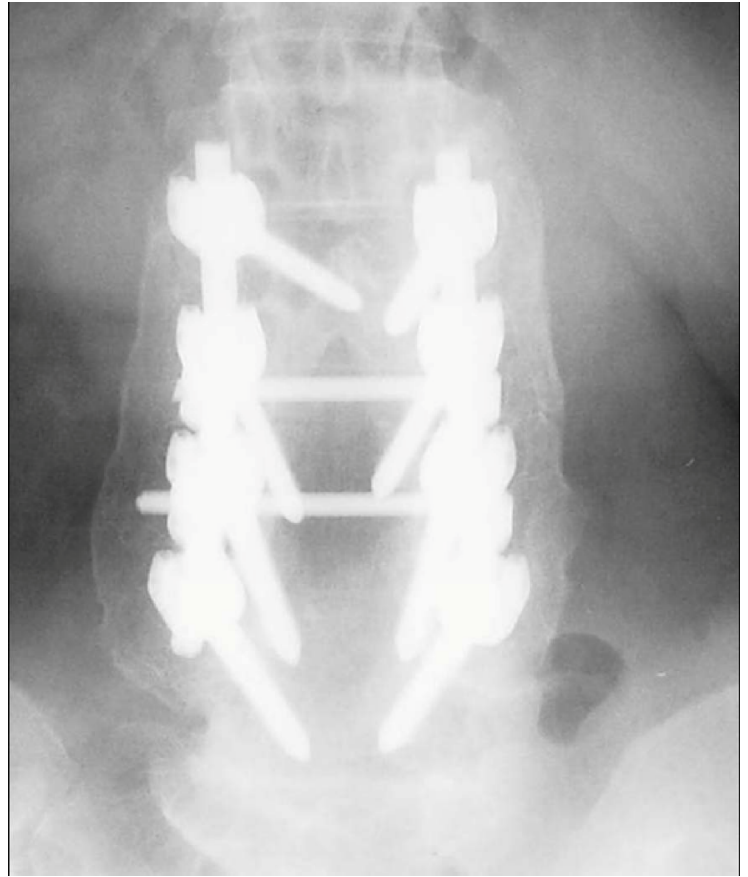

Figure 3. An anteroposterior radiograph at two-year follow-up demonstrated a solid posterolateral fusion. Both sides were graded as "Fusion" and the sizes of the fusion mass were compared.

mental side, and 39 (86.7\%) patients showed solid fusion on the control side. The status of fusion was determined by the radiographic grading system adapted from Christensen study, which stated that if a continuous intertransverse bony bridge was presented on at least one of the two sides then "Fusion" was indicated at all intended levels [12]. The overall fusion rate in this study was $86.7 \%$, and patient data was displayed in Table 1 .

Measuring the concordance between the two sides, kappa coefficient of agreement was calculated as 0.436 and $\mathrm{p}$ value was less than 0.001 , which indicated that there was a statistically significant relationship between the fusion rates of the experimental and control sides (Table 2). Comparing to the control side, the fusion rate of the experimental side is significant decreased $(p=$ 0.014) as shown in Table 3. The odds of fusion for the experimental side was 0.279 times the odds for the control side, and it was statistically significant at alpha 0.05 $(p<0.001)$ in Table 4.

Thirty-eight patients received three-level fusion: L1L4 (2 cases), L2-L5 (28 cases) and L3-S1 (8 cases); 25 (65.8\%) patients showed fusion on the experimental side, and $34(89.5 \%)$ patients showed solid fusion on the control side. In addition, seven patients received four-level fusion: L1-L5 (6 cases) and L2-S1 (1 case); 4 (57.1\%) patients showed fusion on the experimental side and 5 (71.4\%) patients showed solid fusion on the control side. 
Using Fisher's exact test to compare the fusion rate of three-level fusion to four-level fusion, no statistically significant difference was found (Table 5).

Moreover, lumbosacaral spinal fusion was performed in nine cases. The fusion rate was 55.6\% (5/9) for the experimental side and $88.9 \%(8 / 9)$ for the control side. There was also no statistically significant difference for fusion rate when comparing multilevel lumbar fusion to multilevel lumbosacral spinal fusion (Table 6).

Table 1. Patient data.

\begin{tabular}{|c|c|c|c|c|c|c|c|}
\hline Case no. & Age & Sex & Instrumentation System ${ }^{*}$ & Fusion level & Levels & Experimental side & Control side \\
\hline 1 & 56 & F & $\mathrm{RF}$ & L1-L4 & 3 & Fusion & Fusion \\
\hline 2 & 61 & $\mathrm{~F}$ & $\mathrm{RF}$ & L1-L4 & 3 & Fusion & Fusion \\
\hline 3 & 49 & $\mathrm{~F}$ & $\mathrm{RF}$ & L2-L5 & 3 & Fusion & Fusion \\
\hline 4 & 51 & $\mathrm{~F}$ & $\mathrm{RF}$ & L2-L5 & 3 & Non-Fusion & Fusion \\
\hline 5 & 51 & $\mathrm{~F}$ & $\mathrm{RF}$ & L2-L5 & 3 & Non-Fusion & Non-Fusion \\
\hline 6 & 51 & $\mathrm{~F}$ & $\mathrm{RF}$ & L2-L5 & 3 & Non-Fusion & Fusion \\
\hline 7 & 55 & $\mathrm{~F}$ & RF & L2-L5 & 3 & Non-Fusion & Fusion \\
\hline 8 & 55 & $\mathrm{~F}$ & $\mathrm{RF}$ & L2-L5 & 3 & Fusion & Fusion \\
\hline 9 & 59 & $\mathrm{~F}$ & $\mathrm{CD}$ & L2-L5 & 3 & Fusion & Fusion \\
\hline 10 & 59 & F & $\mathrm{RF}$ & L1-L5 & 4 & Fusion & Fusion \\
\hline 11 & 61 & F & $\mathrm{RF}$ & L1-L5 & 4 & Non-Fusion & Non-Fusion \\
\hline 12 & 62 & F & $\mathrm{CD}$ & L2-L5 & 3 & Fusion & Fusion \\
\hline 13 & 64 & $\mathrm{~F}$ & $\mathrm{RF}$ & L2-L5 & 3 & Non-Fusion & Fusion \\
\hline 14 & 64 & $\mathrm{~F}$ & $\mathrm{RF}$ & L2-L5 & 3 & Non-Fusion & Fusion \\
\hline 15 & 64 & $\mathrm{~F}$ & $\mathrm{RF}$ & L1-L5 & 4 & Fusion & Fusion \\
\hline 16 & 67 & $\mathrm{~F}$ & Isola & L2-L5 & 3 & Fusion & Fusion \\
\hline 17 & 71 & $\mathrm{~F}$ & $\mathrm{RF}$ & L2-L5 & 3 & Fusion & Fusion \\
\hline 18 & 71 & $\mathrm{~F}$ & $\mathrm{RF}$ & L2-L5 & 3 & Non-Fusion & Fusion \\
\hline 19 & 71 & $\mathrm{~F}$ & Isola & L2-L5 & 3 & Fusion & Fusion \\
\hline 20 & 73 & $\mathrm{~F}$ & $\mathrm{RF}$ & L2-L5 & 3 & Fusion & Fusion \\
\hline 21 & 73 & $\mathrm{~F}$ & $\mathrm{RF}$ & L2-L5 & 3 & Fusion & Fusion \\
\hline 22 & 74 & $\mathrm{~F}$ & RF & L1-L5 & 4 & Fusion & Fusion \\
\hline 23 & 74 & $\mathrm{~F}$ & $\mathrm{RF}$ & L2-L5 & 3 & Fusion & Fusion \\
\hline 24 & 76 & $\mathrm{~F}$ & $\mathrm{RF}$ & L2-L5 & 3 & Non-Fusion & Fusion \\
\hline 25 & 48 & M & $\mathrm{RF}$ & L1-L5 & 4 & Fusion & Fusion \\
\hline 26 & 51 & M & $\mathrm{RF}$ & L2-L5 & 3 & Fusion & Fusion \\
\hline 27 & 58 & M & $\mathrm{RF}$ & L2-L5 & 3 & Non-Fusion & Non-Fusion \\
\hline 28 & 61 & M & $\mathrm{RF}$ & L2-L5 & 3 & Fusion & Fusion \\
\hline 29 & 65 & M & $\mathrm{RF}$ & L2-L5 & 3 & Fusion & Fusion \\
\hline 30 & 66 & M & $\mathrm{RF}$ & L2-L5 & 3 & Fusion & Fusion \\
\hline 31 & 67 & M & $\mathrm{RF}$ & L2-L5 & 3 & Fusion & Fusion \\
\hline 32 & 67 & M & $\mathrm{RF}$ & L2-L5 & 3 & Non-Fusion & Non-Fusion \\
\hline 33 & 67 & M & $\mathrm{RF}$ & L2-L5 & 3 & Fusion & Fusion \\
\hline 34 & 67 & M & Isola & L2-L5 & 3 & Fusion & Fusion \\
\hline 35 & 68 & M & $\mathrm{RF}$ & L1-L5 & 4 & Non-Fusion & Non-Fusion \\
\hline 36 & 74 & M & $\mathrm{CD}$ & L2-L5 & 3 & Fusion & Fusion \\
\hline 37 & 41 & $\mathrm{~F}$ & $\mathrm{RF}$ & L3-S1 & 3 & Non-Fusion & Fusion \\
\hline 38 & 52 & $\mathrm{~F}$ & $\mathrm{RF}$ & L3-S1 & 3 & Fusion & Fusion \\
\hline 39 & 54 & $\mathrm{~F}$ & $\mathrm{RF}$ & L2-S1 & 4 & Non-Fusion & Fusion \\
\hline 40 & 59 & $\mathrm{~F}$ & $\mathrm{RF}$ & L3-S1 & 3 & Fusion & Fusion \\
\hline 41 & 61 & $\mathrm{~F}$ & $\mathrm{RF}$ & L3-S1 & 3 & Non-Fusion & Fusion \\
\hline 42 & 63 & $\mathrm{~F}$ & $\mathrm{RF}$ & L3-S1 & 3 & Fusion & Fusion \\
\hline 43 & 64 & F & $\mathrm{RF}$ & L3-S1 & 3 & Fusion & Fusion \\
\hline 44 & 67 & $\mathrm{~F}$ & Isola & L3-S1 & 3 & Fusion & Fusion \\
\hline 45 & 65 & M & Isola & L3-S1 & 3 & Non-Fusion & Non-Fusion \\
\hline
\end{tabular}

${ }^{*} \mathrm{CD}$ : Cotrel-Dubousset system; RF: Reduction-Fixation system; Isola: Isola system. 
Comparison of Fusion Rates between Autologous Iliac Bone Graft and Calcium Sulfate

Table 2. Measurement of the concordance between the experimental and control sides.

\begin{tabular}{|c|c|c|c|c|c|c|c|}
\hline & & \multicolumn{4}{|c|}{ Control side } & \multirow{3}{*}{$p$} & \multirow{3}{*}{ kappa } \\
\hline & & \multicolumn{2}{|c|}{ Fusion } & \multicolumn{2}{|c|}{ Non-fusion } & & \\
\hline & & $\mathrm{n}$ & $\%$ & $\mathrm{n}$ & $\%$ & & \\
\hline \multirow{2}{*}{ Experimental side } & Fusion & 29 & 74.4 & 0 & 0 & \multirow{2}{*}{$<0.001$} & \multirow{2}{*}{0.436} \\
\hline & Non-fusion & 10 & 25.6 & 6 & 100 & & \\
\hline
\end{tabular}

Table 3. Fusion rates.

\begin{tabular}{|c|c|c|c|c|c|}
\hline & \multicolumn{2}{|c|}{ Experimental side } & \multicolumn{2}{|c|}{ Control side } & \multirow{2}{*}{$p$} \\
\hline & $\mathrm{n}$ & $\%$ & $\mathrm{n}$ & $\%$ & \\
\hline Fusion & 29 & 64.4 & 39 & 86.7 & \multirow{2}{*}{0.014} \\
\hline Non-fusion & 16 & 35.6 & 6 & 13.3 & \\
\hline
\end{tabular}

Table 4. Fusion rates by Generalized Estimating Equations (GEE).

\begin{tabular}{|c|c|c|c|c|c|c|}
\hline \multirow{2}{*}{ Parameter } & \multirow{2}{*}{ B } & \multirow{2}{*}{ SE } & \multirow{2}{*}{ OR } & \multicolumn{2}{|c|}{ 95\% Wald CI for OR } & \multirow{2}{*}{$p$} \\
\hline & & & & Lower & Upper & \\
\hline Experimental side & -1.277 & 0.3809 & 0.279 & 0.132 & 0.588 & 0.001 \\
\hline Control side & 0 & - & 1 & - & - & - \\
\hline
\end{tabular}

B: beta regression coefficient; SE: standard error; OR: odds ratio, the result of beta value (exponential); CI: confidence interval

Table 5. Comparison between 3-level and 4-level fusion.

\begin{tabular}{|c|c|c|c|c|c|c|}
\hline & & \multicolumn{2}{|c|}{ 3-level } & \multicolumn{2}{|c|}{ 4-level } & \multirow{2}{*}{$p$} \\
\hline & & $\mathrm{n}$ & $\%$ & $\mathrm{n}$ & $\%$ & \\
\hline \multirow{2}{*}{ Control side } & Fusion & 34 & 89.5 & 5 & 71.4 & \multirow{2}{*}{0.230} \\
\hline & Non-fusion & 4 & 10.5 & 2 & 28.6 & \\
\hline \multirow{2}{*}{ Experimental side } & Fusion & 25 & 66.8 & 4 & 57.1 & \multirow{2}{*}{0.686} \\
\hline & Non-fusion & 13 & 34.2 & 3 & 42.9 & \\
\hline
\end{tabular}

Table 6. Comparison between lumbar and lumbosacral spine.

\begin{tabular}{|c|c|c|c|c|c|c|}
\hline & & \multicolumn{2}{|c|}{ L-spine } & \multicolumn{2}{|c|}{ S-spine } & \multirow{2}{*}{$p$} \\
\hline & & $\mathrm{n}$ & $\%$ & $\mathrm{n}$ & $\%$ & \\
\hline Control side & Fusion & 31 & 86.1 & 8 & 88.9 & 1 \\
\hline \multirow{2}{*}{ Experimental side } & Fusion & 24 & 66.7 & 5 & 55.6 & \multirow{2}{*}{0.700} \\
\hline & Non-fusion & 12 & 33.3 & 4 & 44.4 & \\
\hline
\end{tabular}

The size of fusion mass on the control side was compared to the experimental sides in 29 cases, in which "Fusion" was graded on both sides. The experimental side showed larger fusion area than the control side in 13 cases; whereas, the rest 16 cases demonstrated smaller fusion area on the experimental side than the control side. There was no statistical significance between the fusion areas of two sides $(p=0.356)$.

\section{Discussion}

Fusion is indicated as soon as the diagnosis of lumbar spinal instability is established [13]. Deguchi et al. [14] demonstrated a retrospective study of 83 consecutive adult patients with isthmic spondylolisthesis and 69 patients underwent pedicle screw instrumentation operation. As a result, an $82 \%$ fusion rate was achieved in singlelevel fusion and a $74 \%$ rate in two-level fusion. Due to the well-established nature of pedicle screw instrumentation and the well-developed surgical technique, the fusion rate approaches $90 \%$ for single-level fusion procedure [15]. However, in some cases, long segment fusion (two or more levels) was proved to achieve greater advantage in correcting scoliotic curvature and coronal imbalance than short fusion (one level fusion). For example, for patients with severe Cobb angle and rotatory subluxation, long fusion should be performed to minimize adjacent segment disease and improve fixation [16]. Few 
studies have examined the fusion rate in long segment fusion. Kornblatt et al. [17] reported the fusion rates of $90 \%-96.5 \%$ for single-level (L5-S1) fusion, $80 \%-85 \%$ for two-level (L4-S1) fusion and $67 \%-75 \%$ for threelevel (L3-S1) fusion; in addition, the pseudarthrosis rate increases as more motion segments are fused, and it may approach $30 \%$ to $40 \%$ for three-level arthrodesis [18]. All studies concluded that the longer the fused segment the lower the fusion rate. Although the use of iliac crest bone graft is the gold standard for lumbar spinal fusion, a large quantity of bone graft is required for multilevel fusion than single-level fusion, thus it is difficult to obtain a sufficient quantity of autologous iliac bone graft. Consequently, the insufficiency of iliac bone graft and the increased possibility of donor site morbidity remain as problems for spine surgeons $[4,5]$.

Various bone-graft substitutes and extenders have been explored for their uses in posterolateral fusion to overcome the limitations of iliac crest bone graft. In 1995, An et al. [6] compared the difference between allograft and autograft in the same individual and concluded that autograft bone was superior to a mixture of autograft and allograft, frozen allograft, and freeze-dried bone in this order. Gibson et al. [19] carried out a randomized control trial involving 69 patients who received instrumented posterolateral lumbar spinal fusion surgery. Twenty-five of 69 patients underwent two-level fusion, and 44 patients underwent single-level posterolateral instrumented fusion. The trial proved that the patients receiving allograft bone achieved similar outcome as those who receiving autograft bone. However, risk of bacterial and viral disease transmission, difficulty of obtaining safe supply of tissue bank, and lower chance of fusion due to lack of living bone cells to stimulate new bone growth are the drawbacks for allograft bone technique.

Recombinant growth factors and cell-based therapies, which can enhance bone formation and improve fusion rate, have also been considered as alternatives to autograft in posterolateral lumbar fusion. Mulconrey et al. [20] proposed a prospective analysis of 98 patients who underwent multilevel spinal fusion with a minimum of 2-year follow-up. The results showed that bone morphogenetic protein (rhBMP-2) could eliminate the necessity for iliac crest bone graft and yield an overall fusion rate of $95 \%$. Again, the efficacy of rhBMP-2 as a substitute for autograft had been demonstrated in the retrospective cohort study by Taghavi et al. [21]. Among 62 patients who underwent instrumented revision posterolateral fusion of 125 levels, an overall fusion rate of $93.5 \%$ was achieved. The use of rhBMP-2 provided excellent fusion rates in both single- and multilevel fusion; moreover, the use of bone marrow aspirate with allograft may be more cost-effective than rhBMP-2, especially in single-level fusion. Through these clinical studies, bone morphogenetic proteins (BMP) have been proved to successfully stimulate spinal fusion equal to or better than autograft, even in long segment fusions, while eliminating the need for harvesting patient's bone tissue, thus avoiding the potential side effects and complications of the bone harvesting procedure.

Certain ceramics and ceramic composites such as hydroxyapatite and tricalcium phosphate are also used as bone graft extender for spinal fusion. Kasai et al. [22] used local bone chips mixed with apatite-and wollastonite-containing glass ceramic (AWGC) granules for twolevel fusion without spinal instrumentation, and found that the overall fusion rate was approximately $80 \%$. Fujibayashi et al. [23] conducted a retrospective observational study in which single-level posterolateral fusion was performed in 32 patients (19 men and 13 women) who were diagnosed with degenerative spondylolisthesis, isthmic spondylolisthesis, disk herniation with instability and canal stenosis. Local morselized bone harvested from the decompressive site was mixed with hydroxyapatite and beta-tricalcium phosphate (HAP-TCP) granules and sticks and used in the posterolateral lumbar spinal fusion. Although the HAP-TCP treatment produced a smaller fusion mass than autograft, the findings of clinical improvement and solid fusion suggested that this technique was effective. Moreover, the efficacies of these materials have been demonstrated in long segment fusions. A series of 53 patients, undergoing multilevel instrumented fusions (average 7.5 levels) with iliac crest autograft supplemented with beta-tricalcium phosphate (B-TAP), was analyzed in Epstein [24] study. The author concluded that using autograft with an artificial bone graft expander, B-TAP, showed comparable results to those with allograft bone graft expander, demineralized bone matrix (DBM). High fusion rates from both X-ray and $\mathrm{CT}$ evaluations and improved neurological outcomes were achieved while avoiding the inherent risks of allograft use, including infection, allergic and immunologic reactions. In addition, Chang et al. [25] compared the preliminary outcomes of 45 patients who performed multilevel fusions with either cancellous bone or hydroxyapatite graft of 109 degenerative disc levels. Based on the data, they suggested that hydroxyapatite was an effective substitute for cancellous bone marrow, and this osteoconductive material could easily achieve shorter operative times, similar fusion rate and fine postoperative outcome.

Calcium sulfate is another ceramic-based bone graft, which also has been used in spinal fusion and its efficacy of calcium sulfate has been reported in numerous studies. Chen et al. [26] organized a prospective study of 74 patients who received short segment (one or two levels) 
spinal fusion to compare the fusion rate of calcium sulfate pellets plus laminectomy bone chips with fresh autologous iliac bone graft. The results showed equivalent fusion rate and fusion size between these two materials, which suggested the role of calcium sulfate as a bone graft extender in short segment spinal fusion. Another study was conducted to use autogenous bone chips from laminectomy extended with OsteoSet (calcium sulfate) for posterolateral lumbar fusion instead of using bone graft from iliac crest. The clinical results of 124 patients who underwent posterior decompression and posterolateral fusion with instrumentation seems to be promising with the overall union rate of $91 \%$, which indicated that this graft material of bone chips from laminectomy and calcium sulfate is reliable and effective to replace the use of iliac bone graft [27].

In this study, the efficacy of OsteoSet (calcium sulfate) as bone graft substitute was analyzed by comparing to autologous bone graft in multilevel fusion. It has been classified as an alphahemihydrate, which acts primarily as an osteoconductive material that completely resorbs and by newly formed bone, and it also restores anatomic features and structural properties [9]. OsteoSet allows the ingrowth of blood vessels and osteogenic cells, and is resorbed in aqueous media without generating any dissolution products. The potential uses of calcium sulfate bone graft include filling bone defects and expanding grafts by acting as an osteoconductive matrix. In a prospective, nonrandomized, multicenter study by Kelly et al. [11], OsteoSet, surgical grade calcium sulfate pellets, were used in place of morselized cancellous bone graft for treating 109 patients with bone defects who generally required grafting secondary to trauma, periprosthetic bone loss, tumor, or fusion. The radiographic results showed that $99 \%$ of the calcium sulfate had been resorbed and $88 \%$ of the defect area was filled with trabeculated bone at six months postoperatively, which proved that calcium sulfate pellets are considered a convenient, safe and readily available bone graft substitute with successful results.

Alexander et al. [10] reported a preliminary result of 40 patients with degenerative disc disease or spondylolisthesis. The patients received one or two levels of posterolateral lumbar or lumbosacral spinal fusion with spinal canal decompression. Decompression bone plus calcium sulfate pellets were placed on one randomly selected side, and an equal volume of autologous posterior iliac crest bone was placed on the contralateral side. The authors claimed that calcium sulfate pellets plus decompression bone provided equivalent bone formation as autologous iliac crest bone and provide a possible alternative graft material for spinal fusion. However, no previous work has evaluated any similar comparison in long segment (three or more levels) posterolateral lumbar spinal fusion.

This study demonstrated a $64.4 \%(29 / 45)$ fusion rate for using OsteoSet pellets with bone chips from laminectomy on the experimental side, which was equivalent to other multilevel studies. There were 38 patients received three-level fusion, and the fusion rate was $65.8 \%(25 / 38)$ for the experimental side, comparing to a $57.1 \%(4 / 7)$ fusion rate for the remaining seven patients who received four-level fusion. The possibility of pseudoarthrosis is correlated with the extent of fusion levels, and therefore fusion involving more levels (longer segment) would result in higher pseudoarthrosis rate, which is consistent to our result. Furthermore, the fusion rate of autologous iliac bone graft was $86.7 \%(39 / 45)$. In a normal practice without using bone graft substitutes, iliac crest bone graft usually was divided equally into two sides; however, it was only placed on one side in this study, which may explain the high fusion rate achieved by autograft.

For 29 cases in which solid fusion was observed on both sides, the sizes of the fusion mass were compared between the two sides. No statistical significance was found between the fusion areas of two sides. The overall fusion rate in this study was $86.7 \%$, which higher than the multilevel fusion rates that have previously reported. The limitations of this study are the fusion stuatus was only assessed by X-rays and there was no functional outcome of patients. The simplest, cheapest and most commonly used imaging technique for fusion assessments is the plain radiograph; however, other imaging techniques such as computed tomography (CT) scanning may be more appropriate [28]. The use of fine-cut CT scans with sagittal and coronal reconstruction might show better illustration of bony bridging and increase the accuracy of fusion assessments than using plain radiographs $[29,30]$. However, CT scanning is more time and money consuming, and also the radiation exposure to patients is greater since it takes a much greater number of $\mathrm{X}$-ray images. Although CT scans can provide better fusion results, the risks and cost is greater than X-ray, so only plain radiographs were assessed in this study. Another limitation is no functional result of patients was present in this study. For the assessment of fusion, using the functional data seems to more subjective toward patients than using radiographic imaging. In the future, the functional status of patients can be collected and analyze to find out if there is any correlation between the function outcome and the fusion rate for the use of calcium sulfate in multilevel spinal fusion.

In conclusion, the fusion ability of autologous iliac bone graft is higher than calcium sulfate pellets with decompression bone chips in multilevel lumbar spine posterolateral fusion; however, calcium sulfate pellets may 
help expanding the quantity of bone graft with an increased overall fusion rate. Multilevel fusion usually requires a large quantity of bone graft, but the supply of iliac bone graft is usually insufficient. The use of Osteoset pellets combined with bone chips from laminectomy showed increased overall fusion rate in long segment posterolateral spinal fusion while providing equivalent bone formation as autologous iliac crest bone graft, which suggested that calcium sulfate material can be considered as an acceptable bone graft extender in spinal fusion.

\section{Acknowledgements}

We thank Professor Chee-Jee Chang from Graduate Institute of Clinical Medical Science in Chang Gung University for his assistance with statistical analyses.

\section{REFERENCES}

[1] C. L. Schnee, A. Freese and L. V. Ansell, "Outcome Analysis for Adults with Spondylolisthesis Treated with Posterolateral Fusion and Transpedicular Screw Fixation," Journal of Neurosurgery, Vol. 86, No. 1, 1997, pp. 56-63. doi:10.3171/jns.1997.86.1.0056

[2] T. A. Zdeblick, "A Prospective, Randomized Study of Lumbar Fusion. Preliminary Results," Spine (Phila Pa 1976), Vol. 18, No. 8, 1993, pp. 983-991. doi:10.1097/00007632-199306150-00006

[3] C. G. Finkemeier, "Bone-Grafting and Bone-Graft Substitutes," The Journal of Bone \& Joint Surgery, Vol. 84, No. 3, 2002, pp. 454-464. doi: 10.2106/JBJS.K.25334

[4] J. A. Goulet, L. E. Senunas, G. L. DeSilva and M. L. Greenfield, "Autogenous Iliac Crest Bone Graft. Complications and Functional Assessment," Clinical Orthopaedics and Related Research, Vol. 339, No. 339, 1997, pp. 76-81. doi:10.1097/00003086-199706000-00011

[5] E. M. Younger and M. W. Chapman, "Morbidity at Bone Graft Donor Sites," Journal of Orthopaedic Trauma, Vol. 3, No. 3, 1989, pp. 192-195. doi:10.1097/00005131-198909000-00002

[6] H. S. An, K. Lynch and J. Toth, "Prospective Comparison of Autograft vs. Allograft for Adult Posterolateral Lumbar Spine Fusion: Differences among Freeze-Dried, Frozen, and Mixed Grafts," Journal of Spinal Disorders, Vol. 8, No. 2, 1995, pp. 131-135. doi:10.1097/00002517-199504000-00007

[7] S. S. Jorgenson, T. G. Lowe, J. France and J. Sabin, "A Prospective Analysis of Autograft versus Allograft in Posterolateral Lumbar Fusion in the Same Patient. A Minimum of 1-Year Follow-Up in 144 Patients," Spine (Phila Pa 1976), Vol. 19, No. 18, 1994, pp. 2048-2053. doi:10.1097/00007632-199409150-00005

[8] P. J. Nugent and E. G. Dawson, "Intertransverse Process Lumbar Arthrodesis with Allogeneic Fresh-Frozen Bone Graft," Clinical Orthopaedics and Related Research, Vol.
287, 1993, pp. 107-111. doi:10.1097/00003086-199302000-00017

[9] C. G. Finkemeier, "Bone-Grafting and Bone-Graft Substitutes," The Journal of Bone and Joint Surgery. American Volume, Vol. 84, No. 3, 2002, pp. 454-464. doi:10.2106/00004623-200203000-00020

[10] D. I. Alexander, N. A. Manson and M. J. Mitchell, "Efficacy of Calcium Sulfate plus Decompression Bone in Lumbar and Lumbosacral Spinal Fusion: Preliminary Results in 40 Patients," Canadian Journal of Surgery, Vol. 44, No. 4, 2001, pp. 262-266. doi: $10.1503 /$ cjs. 5009328

[11] C. M. Kelly, R. M. Wilkins, S. Gitelis, C. Hartjen, J. T. Watson and P. T. Kim, "The Use of a Surgical Grade Calcium Sulfate as a Bone Graft Substitute: Results of a Multicenter Trial," Clinical Orthopaedics and Related Research, Vol. 382, 2001, pp. 42-50. doi:10.1097/00003086-200101000-00008

[12] F. B. Christensen, M. Laursen, J. Gelineck, S. P. Eiskjaer, K. Thomsen and C. E. Bunger, "Interobserver and Intraobserver Agreement of Radiograph Interpretation with and without Pedicle Screw Implants: The Need for a Detailed Classification System in Posterolateral Spinal Fusion," Spine (Phila Pa 1976), Vol. 26, No. 5, 2001, pp. 538-543. doi:10.1097/00007632-200103010-00018

[13] V. K. Sonntag and F. F. Marciano, "Is Fusion Incidated for Lumbar Spinal Disorders?" Spine (Phila Pa 1976), Vol. 20, No. 24, 1995, pp. 138S-142S. doi:10.1097/00007632-199512151-00011

[14] M. Deguchi. A. J. Rapoff and T. A. Zdeblick, "Posterolateral Fusion for Isthmic Spondylolisthesis in Adults: Analysis of Fusion Rate and Clinical Results," Journal of Spinal Disorders, Vol. 11, No. 6, 1998, pp. 459-464. doi:10.1097/00002517-199812000-00001

[15] S. D. Glassman and J. R. Dimar, "Spinal Instrumentation,” In: K. J. Kowall, Ed., Orthopaedic Knowledge Update, 7th Edition, American Academy of Orthopaedic Surgeons, Rosemont, 2002, pp. 703-716. doi:10.1007/s00264-002-0363-3

[16] K. J. Cho, S. I. Suk, S. R. Park, et al., "Short Fusion versus Long Fusion for Degenerative Lumbar Scoliosis," European Spine Journal, Vol. 17, No. 5, 2008, pp. 650656. doi:10.1007/s00586-008-0615-Z

[17] M. D. Kornblatt, M. P. Casey and R. R. Jacobs, "Internal Fixation in Lumbosacral Spine Fusion. A Biomechanical and Clinical Study," Clinical Orthopaedics and Related Research, No. 203, 1986, pp. 141-150. doi:10.1097/00003086-198602000-00017

[18] J. C. Steinmann and H. N. Herkowitz, "Pseudarthrosis of the Spine," Clinical Orthopaedics and Related Research, Vol. 284, 1992, pp. 80-90. doi:10.1097/00003086-199211000-00011

[19] S. Gibson, I. McLeod, D. Wardlaw and S. Urbaniak, "Allograft versus Autograft in Instrumented Posterolateral Lumbar Spinal Fusion: A Randomized Control Trial," Spine (Phila Pa 1976), Vol. 27, No. 15, 2002, pp. 1599-1603. doi:10.1097/00007632-200208010-00002

[20] D. S. Mulconrey, K. H. Bridwell, J. Flynn, G. A. Cronen 
and P. S. Rose, "Bone Morphogenetic Protein (RhBMP-2) as a Substitute for Iliac Crest Bone Graft in Multilevel Adult Spinal Deformity Surgery: Minimum Two-Year Evaluation of Fusion," Spine (Phila Pa 1976), Vol. 33, No. 20, 2008, pp. 2153-2159. doi:10.1097/BRS.0b013e31817bd91e

[21] C. E. Taghavi, K. B. Lee, G. Keorochana, S. T. Tzeng, J. H. Yoo and J. C. Wang, "Bone Morphogenetic Protein-2 and Bone Marrow Aspirate with Allograft as Alternatives to Autograft in Instrumented Revision Posterolateral Lumbar Spinal Fusion: A Minimum Two-Year Follow-Up Study," Spine (Phila Pa 1976), Vol. 35, No. 11, 2010, pp. 1144-1150. doi:10.1097/BRS.0b013e3181bb5203

[22] Y. Kasai, K. Takegami and A. Uchida, "Mixture Ratios of Local Bone to Artificial Bone in Lumbar Posterolateral Fusion," Journal of Spinal Disorders \& Techniques, Vol. 16, No. 1, 2003, pp. 31-37. doi:10.1097/00024720-200302000-00006

[23] S. Fujibayashi, J. Shikata, C. Tanaka, M. Matsushita and T. Nakamura, "Lumbar Posterolateral Fusion with Biphasic Calcium Phosphate Ceramic," Journal of Spinal Disorders, Vol. 14, No. 3, 2001, pp. 214-221. doi:10.1097/00002517-200106000-00005

[24] N. E. Epstein, "Efficacy of Posterior Cervical Fusions Utilizing an Artificial Bone Graft Expander, Beta Tricalcium Phospate," Surgical Neurology International, Vol. 2, 2011, p. 15. doi:10.4103/2152-7806.76458

[25] W. C. Chang, H. K. Tsou, W. S. Chen, C. C. Chen and C. C. Shen, "Preliminary Comparison of Radiolucent Cages Containing Either Autogenous Cancellous Bone or Hydroxyapatite Graft in Multilevel Cervical Fusion," Jour- nal of Clinical Neuroscience, Vol. 16, No. 6, 2009, pp. 793-796. doi:10.1016/j.jocn.2008.08.034

[26] W. J. Chen, T. T. Tsai, L. H. Chen, et al., "The Fusion Rate of Calcium Sulfate with Local Autograft Bone Compared with Autologous Iliac Bone Graft for Instrumented Short-Segment Spinal Fusion," Spine (Phila Pa 1976), Vol. 30, No. 20, 2005, pp. 2293-2297. doi:10.1097/01.brs.0000182087.35335.05

[27] C. L. Chen, C. L. Liu, S. S. Sun, P. Y. Han, C. S. Lee and W. H. Lo, "Posterolateral Lumbar Spinal Fusion with Autogenous Bone Chips from Laminectomy Extended with OsteoSet," Journal of the Chinese Medical Association, Vol. 69, No. 12, 2006, pp. 581-584. doi:10.1016/S1726-4901(09)70333-8

[28] D. Siambanes and S. Mather, "Comparison of Plain Radiographs and CT Scans in Instrumented Posterior Lumbar Interbody Fusion," Orthopedics, Vol. 21, No. 2, 1998, pp. 165-167. doi:10.3928/9507268

[29] J. R. Dimar, S. D. Glassman, K. J. Burkus and L. Y. Carreon, "Clinical Outcomes and Fusion Success at 2 Years of Single-Level Instrumented Posterolateral Fusions with Recombinant Human Bone Morphogenetic Protein-2/ Compression Resistant Matrix versus Iliac Crest Bone Graft," Spine (Phila Pa 1976), Vol. 31, No. 22, 2006, pp. 2534-2539. doi:10.1097/01.brs.0000240715.78657.81

[30] L. Y. Carreon, S. D. Glassman and M. Djurasovic, "Reliability and Agreement between Fine-Cut CT Scans and Plain Radiography in the Evaluation of Posterolateral Fusions," The Spinal Journal, Vol. 7, No. 1, 2007, pp. 39-43. doi:10.1016/j.spinee.2006.04.005 